\title{
Nanostructures for Medicine and Pharmaceuticals
}

\author{
Xing-Jie Liang, ${ }^{1}$ Anil Kumar, ${ }^{1}$ Donglu Shi, ${ }^{2,3}$ and Daxiang Cui ${ }^{4}$ \\ ${ }^{1}$ CAS Key Laboratory of Biomedical Effects of Nanomaterial and Nanosafety, National Center for Nanoscience and \\ Technology of China, Beijing, China \\ ${ }^{2}$ The Institute for Advanced Materials and Nano Biomedicine, Tongji University, Shanghai 200092, China \\ ${ }^{3}$ School of Electronic and Computing Systems, College of Engineering and Applied Science, \\ University of Cincinnati, Cincinnati, OH 45221, USA \\ ${ }^{4}$ Institute of Micro/Nano Science and Technology, Shanghai Jiao Tong University, Shanghai, China \\ Correspondence should be addressed to Xing-Jie Liang, liangxj@nanoctr.cn
}

Received 22 March 2012; Accepted 22 March 2012

Copyright (c) 2012 Xing-Jie Liang et al. This is an open access article distributed under the Creative Commons Attribution License, which permits unrestricted use, distribution, and reproduction in any medium, provided the original work is properly cited.

The rapid developments in nanostructured materials and nanotechnology will have profound impact in many areas of biomedical applications including delivery of drugs and biomolecules, tissue engineering, detection of biomarkers, cancer diagnosis, cancer therapy, and imaging. This field is expanding quickly, and a lot of work is ongoing in the design, characterization, synthesis, and application of materials, for controlling shape and size at nanometer scale to develop highly advanced materials for biomedical application and even to design better pharmaceutical products. In recent years, novel nanostructure with multifunctionalities has been focused on the use of nanostructures toward solving problems of biology and medicine.

The main scope of this special issue is to demonstrate the latest achievement of nanotechnology and its application in nanomedicine particularly in new approaches for drug delivery such as targeted drug delivery system, nanostructure for drug storage, nanomaterials for tissue engineering, medical diagnosis and treatment, and generation of new kinds of materials from biological sources. Therefore, many critical issues in nanostructured materials, particularly their applications in biomedicine, must be addressed before clinical applications. This special issue devotes several review and research articles encompassing various aspects of nanomaterials for medicine and pharmaceuticals.

We have invited colleagues from worldwide who have been exploring their research in biomedical applications of nanomaterials for design of medicine and pharmaceuticals.

The paper by S. M. Christensen et al. focuses on the applications of various nanostructures and nanodevices in clinical diagnostics and detection of important biological molecules. They have introduced some basic techniques of micro-/nanoscale fabrication that have enabled reproducible production of nanostructures. In the same section, the paper by A. Kumar et al. has broadly mentioned the significant properties of gold playing an important role for the diagnosis of cancer and HIV. S. Jin et al. emphasized the application of quantum dots in biological imaging, and $\mathrm{H}$. Yim et al. mentioned magnetic-resonance-imaging- (MRI-) based contrast agents and multifunctional materials for diagnosis and therapy.

The following section covers the nanomaterials used for pharmaceutical drug delivery and tissue engineering in which C.-W. Li et al. investigated the self-emulsifying drug delivery systems (SEDDSs). Another author has synthesised the starch-chitosan hydrogel, prepared by using the oxidation method, while some papers discussed polybutylcyanoacrylate for oral delivery. One paper mentioned PMAA- (poly(methacrylic acid)-) coated gelatin nanoparticles encapsulated with fluorescent dye for cell imaging. Some papers discuss clay-based polyurethane nanocomposite as local triamcinolone acetonide delivery system. J. Ali et al. mentioned "Fabrication of coated-collagen electrospun PHBV nanofiber film by plasma method and its cellular study." Z. Yang et al. have investigated optimization and preparation of amphotericin B Cubosomes for an oral delivery. In the same section, some of papers discuss the delivery of siRNA and its current challenges for cancer therapy. P. He et al. mentioned toxicity of $\mathrm{TiO}_{2}$ nanopowder, and another paper discusses "Mechanical properties of chitosan-starch composite 
filled hydroxyapatite micro- and nanopowders for biological applications." $\mathrm{F}$. Li et al. investigated the gene delivery in drug-resistant A2780/DDP ovarian cancer cell line via magnetofection.

In another session, X. Junzheng et al. mentioned "Biodistribution study of ${ }^{60} \mathrm{Co}-\mathrm{Co}$ graphitic-shell nanocrystals in vivo." X. Cheng et al. studied about "Characterization of multiwalled carbon nanotubes dispersing in water and association with biological effects," and C. Riggio et al. comprehensively described the nanooncology and its clinical applications for cancer therapy. In the final section, Z.-S. Chen et al. mentioned the synthesis of nanoparticles from microorganisms and their applications, W. Zhang et al. studied "The effect of superhydrophobic surface of titanium on Staphylococcus aureus adhesion," and Th. S. Dhahi et al. demonstrated the "Fabrication of lateral polysilicon gap of less than $50 \mathrm{~nm}$ using conventional lithography."

In summary, the development of novel nanoplatform for the diagnosis and treatment of disease would continue to remain an area of great attention in the field of nanomedicine. In this special issue, we do hope some covered aspects will also provide some interesting information to the readers and researchers to design better pharmaceutical products for human welfare.

\title{
Acknowledgments
}

The Editors would like to appreciate all the authors and coauthors of these papers comprising this special issue for their scientific and research contribution. Moreover, the editors would like to express their thanks to all the reviewers for their time and dedication. We hope that this special issue will attract a wide range of readers/researchers who are working or will join this challenging and fast-developing field.

\author{
Xing-Jie Liang \\ Anil Kumar \\ Donglu Shi \\ Daxiang Cui
}



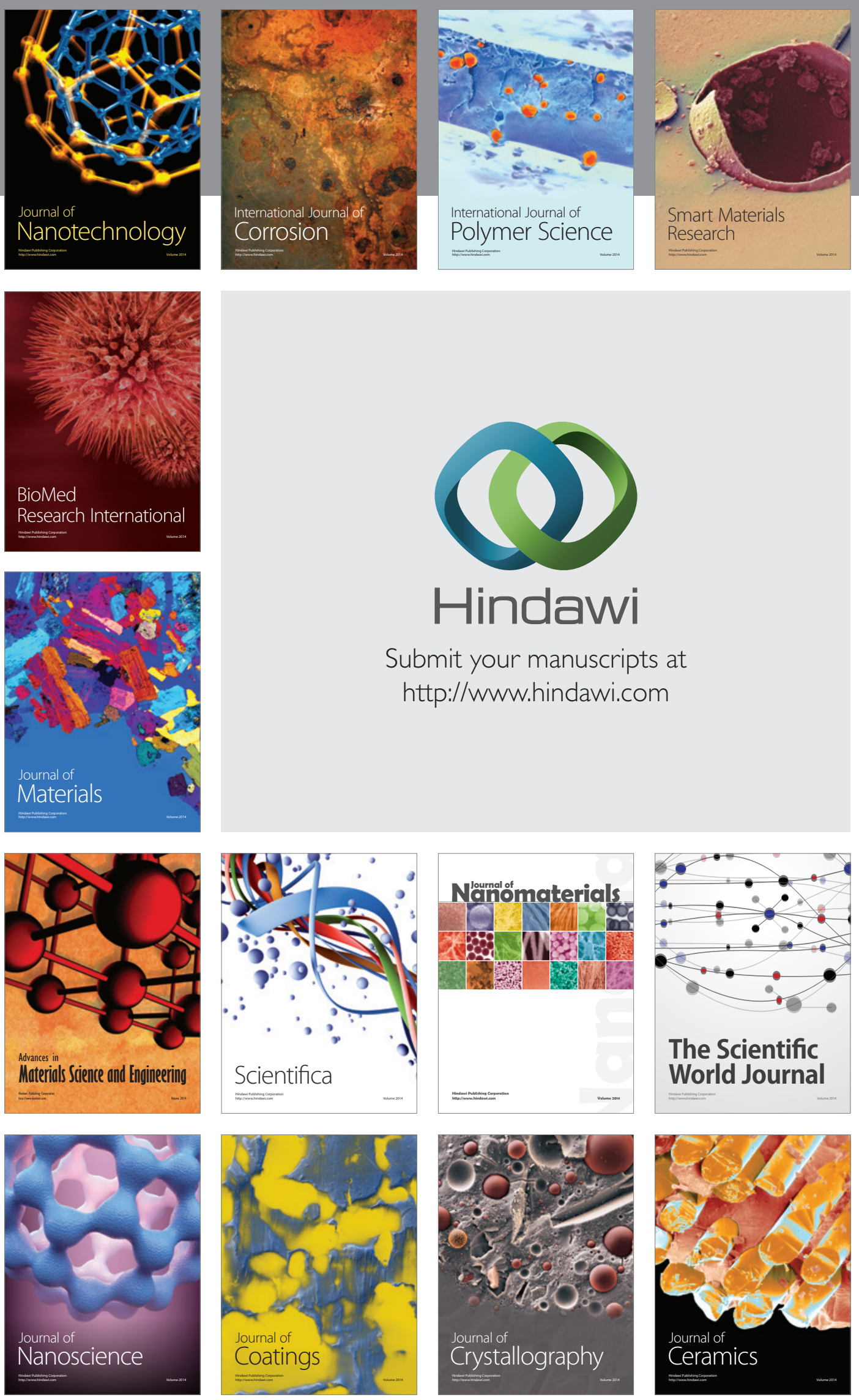

The Scientific World Journal

Submit your manuscripts at

http://www.hindawi.com

\section{World Journal}

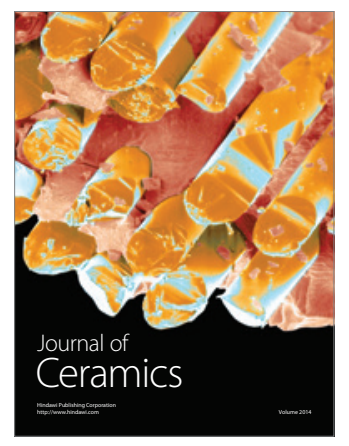

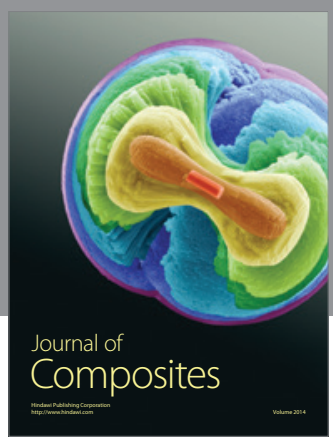
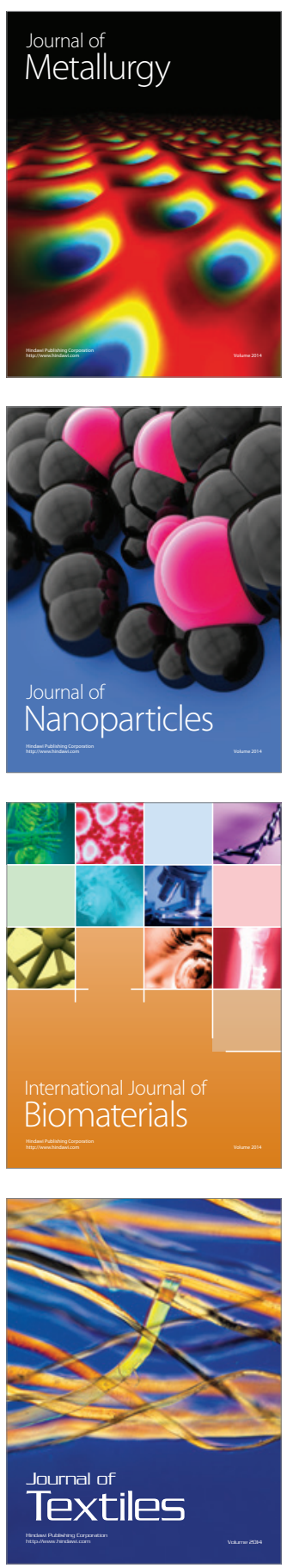